\title{
Concepts of safety reporting
}

\author{
Jan M. Davies, MD • Carmella Steinke, MPA
}

Received: 20 August 2015/Revised: 1 September 2015/Accepted: 11 September 2015/Published online: 28 September 2015

(C) Canadian Anesthesiologists' Society 2015

The historical progression from Imhotep's Edwin Smith papyrus, to Nightingale's studies of Crimean deaths and their causes, through to Gravenstein's mid-1980s description of early "automated anesthesia records"1 parallels the progression in types of reporting systems from hieroglyphics on papyrus, to graphs on paper, through to electronic records. But apart from these functional distinctions, these diverse examples share similar concepts, which, if appropriately applied, can help structure reporting systems.

In this volume of the Journal, Munting et al. describe a reporting system used in a department of anesthesia at a tertiary referral university hospital in Holland. ${ }^{2}$ While this paper illustrates several concepts about reporting of importance for Canadian anesthesiologists, we focus on four:

- Purpose

- Model of the system

- Classification of reports

- Voluntary vs mandatory reporting

J. M. Davies, MD

Department of Anesthesia, Cumming School of Medicine, University of Calgary, Calgary, Canada

J. M. Davies, MD

Department of Psychology, Faculty of Arts, University of

Calgary, Calgary, Canada

J. M. Davies, MD (凹)

Department of Anesthesia, Foothills Medical Centre, Room C-229, 1403 - 29th Street NW, Calgary, AB T2N 2T9, Canada

e-mail: jdavies@ucalgary.ca

C. Steinke, MPA

Health Quality Council of Alberta, Calgary, AB, Canada
The first concept is the overall purpose for reporting. Munting et al. evaluated their newly installed reporting system to identify topics for improvements in clinical care and patient safety. While both points are important, the latter is essential. The overall purpose for reporting is to ensure that a department or organization receives safetyrelated information that can then be used to manage and improve safety. As well stated by Fiorina, data "must be stored and analyzed and managed and shared, because of course, the goal is to transform data into information and information into insight". A

A department/organization with a safety management system will have various sources of data, such as reports from personnel and administrative databases. Although reports from personnel represent only one source of information, they play a vital role in helping to improve care. We agree with Dutton's statement in his recent editorial describing the Anesthesia Quality Institute: "All quality improvement is local". ${ }^{3}$ Improvements in care need to be undertaken relatively closely to the site of the event. Analyzing reports, implementing recommended changes, and providing feedback that changes were initiated from information garnered from reports, will help change the attitudes and subsequent behaviours of personnel involved. ${ }^{4}$ Furthermore, this information can then be distributed to other centres, even worldwide. And as the attitudes and behaviours change, so will the culture - the unspoken but clearly perceived "way we do things around here". 5 A department/organization in which individuals feel free to report safety-related information, knowing that

\footnotetext{
A Fiorina $C$. Information: the currency of the digital age. Speech given at Oracle OpenWorld, San Francisco, 6 December 2004. Available from URL: http://www.hp.com/hpinfo/execteam/speeches/ fiorina/04openworld.html (accessed September 2015).
} 
they, as messengers, will not be "shot", will be on its way towards developing a maturing safety culture. ${ }^{6}$ For example, Fasting and Gisvold described the "culture of openness" in their department, where meetings to discuss reports were met with "enthusiasm from both doctors and nurses". 7 A department/organization with a stronger safety culture is one that trains reporters to be proactive in seeking safety deficiencies and rewards them for so doing. ${ }^{6}$ Hence, safety inadequacies can be dealt with even before a close call.

This is essentially what Munting et al. established in 2005 - i.e., a pattern of reporting that would identify their department as having a developing safety culture. ${ }^{6}$ Three months after implementing an anesthesia information management system (AIMS), they asked anesthesiologists and anesthesia residents to submit reports of "critical incidents" (CIs) through the AIMS. A critical incident was defined as "an event that could have led (if not discovered or corrected in time) or did lead to an undesirable outcome, i.e., ranging from increased length of hospital stay to death or permanent disability". This is essentially the definition first offered by Jeff Cooper et al. in their 1978 landmark publication on human factors and preventable anesthesia mishaps. ${ }^{8}$ Reports could be related to process of care, such as "kinking of [the endotracheal] tube", or to outcome, such as "anemia".

While it is important to report those rare but important outcomes, such as death (which Munting et al. did not list in their Tables 4 or 6 ), clearly defining process events will also encourage reporting close calls. It is vital to learn about these more common events: the patient was not harmed, personnel were not upset by the events, and the organization did not have to deal with the aftermath. In addition, staff reporting close calls can be asked to answer the essential question, "What saved the day?", and to suggest recommendations for improvements to the system. ${ }^{9}$

Having a safety management system with a clearly defined map of its territory - a model of the system - can facilitate searching for CIs (that can then be considered as markers for underlying system deficiencies). Just as travellers without a map can be surprised by their journey and their destination, those who manage safety require a model to help minimize safety surprises and deal with them effectively when they do arise. A system model should drive not only the safety management system but also the structure of the databases from which safety information is derived, and thus a system's classification of incidents ${ }^{10}$ and even the terminology used.

The Dutch reports outlined by Munting et al. were initially classified by the reporting anesthesiologists using a 20 -item list (established by the Netherlands Society of
Anesthesiologists). This list included a "Miscellaneous" category, which suggests incomplete modelling of the classification of incidents. Just as maps lack a place called "Other", similarly, classification systems should not include that imprecise term. Indeed, nearly $22 \%$ of the CIs were assigned to "Miscellaneous". One of the team members then reclassified all reports using a separate 60item list from the German Board of Anesthesiology, plus 35 additional items. Even this expansion was found to be insufficient, with "Miscellaneous" - although now with sub-categories - still comprising "other" reports.

We do question why certain CIs, e.g., hypotension, were included in the classification. Some of these CIs are transient physiological states that cannot be classified without supplementary details. ${ }^{11}$ Rather than have anesthesiologists report such CIs, why not choose the physiologic changes worth studying and collect data from the AIMS? Anesthesiologists could then be encouraged to report technical difficulties or "problems" that are "encountered by everyone" 7 and to answer the question, "Did the patient suffer or nearly suffer harm?"

Third, the type of classification of reports Munting and co-workers chose to use can be described as front- rather than back-end. Typically front-end classification requires reporters to provide specific information using (multiple) drop-down and tick boxes, not only to specify what happened and how but also to classify what was reported. Reports requiring front-end classification can help with the collection of details that might otherwise be omitted through the use of triggering questions. But busy clinicians can find this type of system frustrating to use because of the time often needed to complete a complicated electronic report. There is also the possibility that a reporter's proximity to the incident might lead to a focus on person-specific details, often described as "human factors". This may result in an underestimation of other contributing factors in the system, for example, those related to the organization and/or regulators. At the other end of the spectrum is the requirement for only a simple narrative - i.e., telling the story without classification. The simplicity of the narrative encourages detailed reporting ${ }^{4}$ and classification is done at the back or receiving end. Nevertheless, because free text can make after-report coding and classifying more challenging, this task is best performed by groups of specialized safety staff. ${ }^{9}$

The fourth concept concerns mandatory vs voluntary reporting. While aviation has many rules defining what must be reported, there are few similar requirements in healthcare and even fewer in anesthesia. For example, at the regulatory level of the healthcare system, a death that occurs under anesthesia or during recovery from anesthesia must be reported to a medical examiner or 
coroner in only seven of Canada's provinces and territories. $^{\mathrm{B}, \mathrm{C}, \mathrm{D}, \mathrm{E}, \mathrm{F}, \mathrm{G}, \mathrm{H}}$

Munting et al. chose a voluntary system, the benefit being that people can choose to report and their willingness to do so can be influenced by whether or not the system provides anonymous or de-identified confidential reporting. The former offers reassurance to those who may be worried about repercussions for their actions, or even their reports. The latter can provide similar reassurance if the reporting system is securely separated from any disciplinary or regulatory function. Confidential reporting also allows safety personnel to contact the reporter for further contextual details. In fact, Munting et al.'s system was neither anonymous nor confidential.

Apart from any "must vs could" differentiation, the optional nature of voluntary reporting systems, especially those that are not confidential, means that they are subject to both underreporting and what we term as "enthusiastic" reporting. Munting et al. noted that, during January 2005 to May 2011, 65,985 patients received 110,310 anesthetics that generated 3,904 voluntary reports of critical incidents from 3,807 separate anesthetics. Their overall report completion rate of more than $94 \%$ was particularly laudable and attributed to reminders given in the AIMS during skin closure, by e-mail after completion of the anesthetic, and by feedback at regular meetings. Interestingly, $6 \%$ of cases had no reports submitted and some anesthesiologists reported more than others. One anesthesiologist contributed 288 reports, and data shown in

\footnotetext{
B Province of Alberta. Fatality Inquiries Act. Revised Statutes of Alberta 2000. Chapter F-9. Current as of December 17, 2014. Available from URL: http://www.qp.alberta.ca/documents/Acts/F09. pdf (accessed September 2015).

${ }^{C}$ Province of Manitoba. C.C.S.M. c F52. The Fatality Inquiries Act. Available from URL: https://web2.gov.mb.ca/laws/statutes/ccsm/ f052e.php (accessed September 2015).

D Province of Newfoundland \& Labrador. SNL1995 Fatalities Investigations Act. Chapter F-6.1. An Act Respecting the Investigation of Fatalities. Available from URL: http://www. assembly.nl.ca/legislation/sr/statutes/f06-1.htm (accessed September 2015).

E Northwest Territories. Coroners Act. RSNWT (Nu) 1988, c C-20. Available from URL: https://www.canlii.org/en/nt/laws/stat/rsnwt1988-c-c-20/latest/rsnwt-1988-c-c-20.html (accessed September 2015).

F Province of Nova Scotia. Fatality Investigations Act. Chapter 31 of the Acts of 2001. Available from URL: http://nslegislature.ca/legc/ statutes/fatality\%20investigations.pdf (accessed September 2015).

G Nunavut. Coroners Act. RSNWT (Nu) 1988, c C-20. Available from URL: https://www.canlii.org/en/nu/laws/stat/rsnwt-nu-1988-c-c20/latest/rsnwt-nu-1988-c-c-20.html (accessed September 2015).

$\mathrm{H}$ Province of Prince Edward Island. Chapter C-25.1. Coroners Act. Available from URL: http://www.gov.pe.ca/law/statutes/pdf/c-25_1. pdf
}

their Table 3 were limited to those reports where no more than $15 \%$ of the events were from one anesthesiologist.

A note of caution is warranted in interpreting these sorts of data - specifically, because the true number of events will be unknown, and therefore, the number of reports cannot be used as an accurate numerator for calculating complication rates or for determining the frequency of specific reported events. Thus, inferential statistical analysis should not be performed using data from voluntary reporting systems. Similarly, information from voluntary reporting systems cannot be used to monitor safety with accuracy (i.e., the frequency and severity of safety events). Demonstration of "trends" in the number of types of voluntarily reported incidents should be interpreted to mean that only the number of reports is changing and not that one aspect of care has improved or worsened.

In summary, the work of Munting et al. provides some useful concepts for Canadian anesthesiologists, not the least of which is that implementing a safety reporting system through AIMS can offer a key source of safety information to guide improvement activities. Implementing a robust and well-structured reporting system is an important step in improving the safety of our patients.

\section{Concepts des divulgations de sécurité}

Il existe un parallélisme historique entre la progression constatée depuis les écrits d'Imhotep (le papyrus Edwin Smith) jusqu'aux études sur les décès et leurs causes par Florence Nightingale en Crimée, en passant par les descriptions des premiers «dossiers automatisés d'anesthésie » par Gravenstein ${ }^{1}$ au milieu des années 1980, avec la progression des différents types de systèmes de divulgations qui sont passés des hiéroglyphes sur papyrus, aux graphiques sur papier et maintenant aux dossiers électroniques. Mais, hormis ces distinctions fonctionnelles, ces divers exemples ont en commun des concepts similaires qui peuvent contribuer à structurer les systèmes de divulgations s'ils sont correctement appliqués.

Munting et coll. décrivent dans ce numéro du Journal un système de divulgations utilisé dans un département d'anesthésie d'un hôpital universitaire de référence aux Pays-Bas. $^{2}$ Leur article illustre plusieurs concepts importants pour les anesthésiologistes canadiens concernant la nature des divulgations, mais nous nous concentrerons sur quatre d'entre eux :

- le but des divulgations,

- le modèle de système, 
- la classification des divulgations,

- leur caractère volontaire ou obligatoire.

Le premier concept est général : le but des divulgations. Munting et coll. ont évalué leur système de divulgations nouvellement installé pour identifier des sujets d'amélioration des soins cliniques et de la sécurité des patients. Bien que ces deux points soient importants, le dernier est essentiel. L'objectif global des divulgations est de veiller à ce qu'un département ou une organisation reçoive une information ayant trait à la sécurité qui puisse alors être utilisée pour gérer et améliorer la sécurité. Comme l'a bien dit Fiorina, les données « doivent être sauvegardées et analysées, gérées et partagées, parce que le but est évidemment de transformer ces données en information et cette information en perspective ». ${ }^{\mathrm{A}}$

Un département ou une organisation disposant d'un système de gestion de la sécurité aura différentes sources de données, telles que des divulgations du personnel et des bases de données administratives. Même si les divulgations émises par le personnel ne représentent qu'une source d'informations parmi d'autres, ils jouent un rôle crucial dans l'amélioration des soins. Nous partageons l'avis de Dutton dans son récent éditorial lorsqu'il décrit l'Anesthesia Quality Institute (Institut pour la qualité de l'anesthésie): "toute amélioration de la qualité est locale ». ${ }^{3}$ L'amélioration des soins doit être entreprise relativement près du site de l'évènement. L'analyse des divulgations, la mise en ouvre des changements recommandés et la fourniture d'un retour d'information à l'origine de changements ont commencé à partir des renseignements colligés dans les divulgations; cela contribuera au changement d'attitudes et de comportements ultérieurs des personnels concernés. ${ }^{4}$ En outre, ces renseignements peuvent être communiqués à d'autres centres, voire au monde entier. Et, pendant que les attitudes et les comportements changent, la culture changera aussi : cette culture non dite mais clairement perçue de « la façon dont nous faisons les choses ici ». ${ }^{5} \mathrm{Un}$ département ou une organisation dans lequel/laquelle les individus se sentent libres de rapporter une information ayant trait à la sécurité, sans être les «messagers à abattre », commencera à bâtir une culture de sécurité mature. ${ }^{6}$ Par exemple, Fasting et Gisvold ont décrit la «culture d'ouverture » qui règne dans leur département et où les réunions destinées à discuter des divulgations ont été accueillies avec « enthousiasme à la fois par les médecins et les infirmières $»{ }^{7}$ Un département ou une organisation qui a une culture de sécurité plus forte est celui/celle qui forme les rapporteurs à être proactifs dans la recherche des lacunes en matière de sécurité et qui les récompense pour le faire. ${ }^{6} \mathrm{En}$ conséquence, les insuffisances en matière de sécurité peuvent être réglées avant de sentir le vent du boulet.
Il s'agit pratiquement de ce que Munting et coll. ont mis en place en 2005 : c'est-à-dire un mode de création et traitement des divulgations qui positionnerait leur département comme ayant une culture de progrès en matière de sécurité ${ }^{6}$ Trois mois après avoir mis en œuvre un système de gestion de l'information en anesthésie (SGIA), ils ont demandé aux anesthésiologistes et aux résidents en anesthésie d'envoyer des rapports sur des « incidents critiques» (IC) via le SGIA. Un incident critique était défini comme étant « un évènement qui aurait pu conduire (s'il n'avait été découvert ou corrigé à temps) ou qui avait conduit à un aboutissement indésirable, allant de l'allongement de la durée d'hospitalisation, au décès, ou à une invalidité permanente du patient ». C'est pour l'essentiel la définition proposée par la première fois par Jeff Cooper et coll. dans leur article de référence de 1978 sur les facteurs humains et les accidents évitables en anesthésie. ${ }^{8}$ Les divulgations pouvaient avoir trait au processus de soins comme une «plicature du tube (endotrachéal)» ou à une conséquence, telle qu'une « anémie ».

Il est bien sûr important de signaler des conséquences rares, mais importantes, telles qu'un décès (ce que Munting et coll. n'avaient pas cité dans leurs tableaux 4 ou 6), mais la définition claire des évènements inclus dans les processus encouragera également le signalement des incidents évités de justesse. Il est vital d'en savoir plus sur ces évènements plus fréquents : le patient n'a pas subi de lésions, le personnel n'a pas été bouleversé par les évènements et l'organisation n'a pas eu à en gérer les conséquences. De plus, on peut demander au personnel signalant des incidents évités de justesse de dire « ce qui a sauvé la mise ce jour-là » et leur demander de suggérer des recommandations pour améliorer le système. ${ }^{9}$

Le fait d'avoir un système de gestion de la sécurité avec une délimitation claire et précise de son domaine de compétence (c'est-à-dire un modèle de système) peut faciliter la recherche d'IC (que l'on peut considérer comme des marqueurs des lacunes sous-jacentes du système). Tout comme des voyageurs sans carte peuvent être surpris par la tournure du voyage et leur destination, ceux qui gèrent la sécurité ont besoin d'un modèle pour contribuer à minimiser les mauvaises surprises et à les gérer efficacement quand elles apparaissent. Un modèle de système devrait non seulement être le moteur du système de gestion de la sécurité, mais aussi de la structure des bases de données d'où proviennent les renseignements sur la sécurité et, par conséquent, une classification des incidents du système ${ }^{10}$ et même de la terminologie utilisée.

Les rapports néerlandais dont parlent Munting et coll. étaient initialement classés par les anesthésiologistes les soumettant au moyen d'une liste de 20 items (établie par la 
Société des anesthésiologistes des Pays-Bas). Cette liste incluait une catégorie «Divers » suggérant le caractère incomplet de la modélisation de la classification des incidents. De même qu'il n'y a pas sur les cartes un lieu marqué « Autre », les systèmes de classification ne doivent pas contenir de termes vagues. Effectivement, près de $22 \%$ des IC étaient attribués à la catégorie « Divers ». Un des membres de l'équipe a recatégorisé tous les rapports en utilisant une liste de 60 items du Conseil allemand des anesthésiologistes et 35 items supplémentaires. Même cette expansion s'est avérée insuffisante car la catégorie «Divers» (disposant pourtant à ce stade de sous-catégories) incluait toujours des rapports « Autres ».

Nous nous demandons pourquoi certains IC, par exemple l'hypotension, ont été inclus dans la classification. Certains de ces IC sont des états physiologiques transitoires qui ne peuvent pas être classés sans détails supplémentaires. ${ }^{11}$ Plutôt que de demander aux anesthésiologistes de signaler de tels IC pourquoi ne pas choisir des modifications physiologiques valant la peine d'être étudiées et collecter les données à partir du SGIA? Les anesthésiologistes pourraient alors être encouragés à rapporter des difficultés techniques ou des 《 problèmes » qui sont « rencontrés par tous » ${ }^{7}$ et invités à répondre à cette question : «le patient en a-t-il subi ou failli subir des conséquences délétères pour sa santé? »

Troisièmement, le type de classification des divulgations que Munting et coll. ont choisi d'utiliser peut être décrit comme un système descriptif frontal plutôt que comme un système de gestion «de l'arrière ». Classiquement, pour une classification de l'avant, les rapporteurs doivent fournir une information spécifique utilisant des listes déroulantes (multiples) et des cases à cocher non seulement pour indiquer ce qu'il s'est passé et comment, mais aussi pour classer ce qui a été signalé. Les divulgations nécessitant une classification de l'avant peuvent contribuer à la collecte de détails par l'emploi de questions stimulantes et qui, autrement, auraient pu être oubliés. Mais les cliniciens à l'emploi du temps chargé peuvent trouver ce type de système frustrant à utiliser en raison du temps souvent nécessaire pour remplir un rapport électronique compliqué. Il est également possible que la proximité de l'incident conduise le rapporteur à se focaliser sur des détails spécifiques à une personne, les décrivant souvent comme des «facteurs humains ». Cela peut se traduire par une sous-estimation des autres facteurs contributifs dans le système, par exemple des facteurs en rapport avec l'organisation et/ou des régulateurs. À l'autre extrémité du spectre, on trouve la demande d'une simple narration, c'est-à-dire le récit de l'évènement sans classification. La simplicité de la narration encourage des divulgations détaillées ${ }^{4}$ et la classification a lieu de l'arrière ou par la partie qui reçoit l'information. Néanmoins, un texte libre peut rendre le codage et la classification a posteriori plus difficiles et cette tâche est donc mieux réalisée par des groupes de personnels spécialisés en sécurité. ${ }^{9}$

Le quatrième concept concerne le caractère obligatoire ou volontaire de la divulgation. Alors qu'il y a dans le domaine aéronautique de nombreuses règles définissant ce qui doit être déclaré, il y a très peu d'exigences similaires dans le domaine des soins de santé et encore moins en anesthésie. Par exemple, sur le plan réglementaire du système des soins de santé, un décès survenant sous anesthésie ou pendant la sortie de l'anesthésie doit être signalé à un médecin légiste ou à un coroner dans seulement sept des provinces et territoires du Canada. ${ }^{\text {B,C,D,E,F,G,H }}$

Munting et coll. ont choisi un système volontaire qui a pour avantage que les intéressés peuvent choisir de faire le signalement et leur désir de le faire peut être influencé par le fait que le système offre (ou pas) la possibilité d'une divulgation anonyme ou confidentielle sans identité. Dans le premier cas, le système permet de rassurer ceux qui seraient inquiets des répercussions de leurs actions ou même de leurs divulgations. Dans le dernier cas, il y a une réassurance semblable si le système de signalement est strictement distinct de toute fonction disciplinaire ou réglementaire. Le signalement confidentiel permet également au personnel chargé de la sécurité de contacter le rapporteur pour avoir des détails complémentaires sur le contexte. En fait, le système de Munting et coll. n'était ni anonyme ni confidentiel.

En dehors d'une alternative « doit» contre «peut», la nature facultative des systèmes de déclaration volontaire, et particulièrement de ceux qui ne sont pas confidentiels, signifie qu'ils sont à la fois soumis à des phénomènes de sous-signalement et de ce que nous appelons des « signalements enthousiastes ». Munting et coll. ont noté que de janvier 2005 à mai 2011, 65985 patients ont reçu 110310 anesthésies qui ont généré 3904 rapports volontaires d'incidents critiques liés à 3807 anesthésiologistes distincts. Leur taux global de remplissage des rapports a été supérieur à $94 \%$, ce qui est particulièrement louable et attribué aux rappels donnés sur le SGIA au cours de la fermeture des plans cutanés, par courriel après la fin de l'anesthésie et par retour d'information au cours de réunions régulières. Il est intéressant de noter qu'aucun rapport n'a été soumis pour $6 \%$ des cas et que certains anesthésiologistes faisaient plus de rapports que d'autres. Un anesthésiologiste a contribué à 288 rapports et les données présentées dans leur Tableau 3 ont été limitées aux rapports où pas plus de $15 \%$ des évènements provenaient d'un seul anesthésiologiste.

Ces sortes de données justifient néanmoins une mise en garde : considérant que le nombre véritable d'évènements 
restera inconnu, le nombre de divulgations ne peut donc pas être utilisé comme numérateur valide pour calculer exactement les taux de complications ou pour déterminer la fréquence des évènements spécifiques rapportés. Ainsi, aucune analyse statistique inférentielle ne doit être effectuée sur ces données provenant de systèmes de déclarations volontaires. Pareillement, l'information tirée des systèmes de déclarations volontaires ne peut pas servir à contrôler la sécurité avec exactitude (c'est-à-dire, la fréquence et sévérité des évènements concernant la sécurité). La mise en évidence de «tendances » dans le nombre de types d'incidents déclarés volontairement ne doit être interprétée que pour signifier que seul le nombre de divulgations change, mais pas pour conclure qu'un aspect des soins s'est amélioré ou aggravé.

En résumé, le travail de Munting et coll. procure quelques concepts utiles aux anesthésiologistes canadiens dont le moindre est que la mise en œuvre d'un système de déclaration sur la sécurité par le biais du SGIA peut offrir une source essentielle de renseignements sur la sécurité pour guider des actions d'amélioration. L'implémentation d'un système de déclarations robuste et bien structuré constitue une étape importante pour l'amélioration de la sécurité de nos patients.

Conflicts of interest None declared.

Conflits d'intérêts Aucun déclaré.

\section{References}

1. Gravenstein JS. The automated anesthesia record. Int J Clin Monit Comput 1986; 3: 131-4.
2. Munting K, van Zaane B, Schouten A, van Wolfswinkel L, de Graaf $J$. Reporting critical incidents in a tertiary hospital: a historical cohort study of 110,310 procedures. Can J Anesth 2015; 62: this issue. DOI: 10.1007/s12630-015-0492-y

3. Dutton RP. Making a difference: the Anesthesia Quality Institute. Anesth Analg 2015; 120: 507-9.

4. Reed S, Arnal D, Frank $O$, et al. National critical incident reporting systems relevant to anaesthesia: a European survey. British Journal of Anaesthesia 2014; 112: 546-55.

5. Bower M. Company philosophy: the way we do things around here. Chapter 2. In: Bower M (Ed.). The Will to Manage Corporate Success Through Programmed Management. NY: The McGraw-Hill Publishing Company; 1966: 22-41.

6. Westrum R. A typology of organisational cultures. Qual Saf Health Care 2004; 13(Suppl II): ii22-7.

7. Fasting S, Gisvold SE. Data recording of problems during anaesthesia: presentation of a well-functioning and simple system. Acta Anaesthesiol Scand 1996; 40: 1173-83.

8. Cooper JB, Newbower RS, Long CD, McPeek B. Preventable anesthesia mishaps: a study in human factors. Anesthesiology 1978; 49: 399-406.

9. Davies JM, Duchscherer C, McRae G. A new reporting system: was the patient harmed or nearly harmed - chapter 7. In: Anca JM Jr (Ed.). Multimodal Safety Management and Human Factors. Crossing the Borders of Medical, Aviation, Road and Rail Industries. Aldershot, UK: Ashgate Publishing Limited; 2007: 61-71.

10. Davies JM. Application of the Winnipeg model to obstetric and neonatal audit. Top Health Inf Manage 2000; 20: 12-22.

11. Schiff JH, Welker A, Fohr B, et al. Major incidents and complications in otherwise healthy patients undergoing elective procedures: results based on 1.37 million anaesthetic procedures. Br J Anaesth 2014; 113: 109-21. 